\title{
Surgical Strategies for Cervical Spinal Neurinomas
}

\author{
Kiyoshi ITO, ${ }^{1}$ Tatsuro AOYAMA, ${ }^{1}$ Yoshinari MIYAOKA, ${ }^{1}$ Tetsuyoshi HORIUCHI, ${ }^{1}$ \\ and Kazuhiro HONGO ${ }^{1}$
}

${ }^{1}$ Department of Neurosurgery, Shinshu University School of Medicine, Matsumoto, Nagano

\begin{abstract}
Cervical spinal neurinomas are benign tumors that arise from nerve roots. Based on their location, these tumors can also take the form of a dumbbell-shaped mass. Treatment strategies for these tumors have raised several controversial issues such as appropriate surgical indications and selection of surgical approaches for cervical dumbbell-shaped spinal neurinomas. In this report, we review previous literature and retrospectively analyze cervical spinal neurinoma cases that have been treated at our hospital. Surgical indications and approaches based on tumor location and severity are discussed in detail. Thus, with advances in neuroimaging and neurophysiological monitoring, we conclude that appropriate surgical approaches and intraoperative surgical manipulations should be chosen on a case-by-case basis.
\end{abstract}

Key words: dumbbell type, nerve root resection, neurofibromatosis, spinal neurinoma, surgical strategy

\section{Introduction}

Spinal neurinomas are benign tumors that account for one-third of all spinal cord tumors. ${ }^{1)}$ Approximately $80 \%$ of these tumors arise from intradural nerve roots, especially the dorsal roots. Sometimes, spinal neurinomas form across the dura mater as a result of extradural growth along the nerve roots, and as a result, form a dumbbell-shaped mass. ${ }^{1-3)}$ These tumors account for around $20 \%$ of all spinal neurinomas.

In contrast to neurofibromas, which arise from nerve fibers, a neurinoma is a tumor that arises from nerve sheath cells. Moreover, neurinomas consist of benign neoplastic Schwann cells, while neurofibromas contain a mix of Schwann cells, perineural-like cells, and fibroblasts interspaced with nerve fibers and myxoid matrices. ${ }^{45}$ However, it has been histologically and clinically difficult to distinguish between these tumor types and they are sometimes described collectively as nerve sheath tumors.

Although nerve sheath tumors are relatively common among spinal cord tumors, treatment strategies for these tumors have raised several controversial issues. Such disputes include appropriate surgical indications for cervical neurinomas, including neurofibromatosis, and selection of surgical approaches for cervical dumbbell-shaped spinal neurinomas.

In this article, we review previous literature and

Received December 3, 2014; Accepted April 30, 2015 retrospectively analyze spinal neurinoma cases that have been treated at our hospital. We specifically focus on the current standard treatment for cervical spinal neurinomas.

\section{Surgical Indications and Treatment of Cervical Spinal Neurinomas}

As the majority of spinal neurinomas cases are benign, surgery is principally indicated for symptomatic tumors. On the other hand, asymptomatic tumors are subject to follow-up using diagnostic imaging modalities, such as magnetic resonance (MR) imaging, and surgery is recommended for tumors that exhibit growth or become symptomatic. However, as described below, neurinomas associated with neurofibromatosis (NF) type 2 are multifocal, grow quickly, and their associated symptoms tend to be more serious. A previous report promoted early surgery because these tumors tend to infiltrate the nerve roots, thus increasing surgical difficulty. $\left.{ }^{6}\right)$ Moreover, these tumors tend to frequently relapse and exhibit multifocality that should be understood when considering surgery for patients with NF type 2 .

\section{Surgical Strategy for Cervical Non-dumbbell-shaped Spinal Neurinomas in the Intradural Region}

Surgical exposure of a cervical spinal neurinoma without facetectomy and spinal instrumentation 
depends on the specific anatomy of the lesion. For example, tumors that are small and intradural can be approached posteriorly, and this approach can be utilized with laminoplastic hemilaminotomy to prevent postoperative subcutaneous fluid collection caused by cerebrospinal fluid (CSF) leakage, kyphotic deformity, and infection. On the other hand, ventrally located tumors may require partial facetectomy. ${ }^{7-12)}$

According to preoperative neuroimages, safe removal of a tumor requires that a wide operative field be maintained so that the entire mass can be confirmed. Furthermore, if a tumor proves to be mobile by changing posture with MR imaging taken some time before surgery, a wider operative field is needed.

When a tumor is located dorsal to the denticulate ligaments, it can be directly separated from the surrounding nerve tissue. However, it is important to identify a tumor if it is located ventral to the denticulate ligaments, or if it has extended to the anterior or anterolateral direction of the spinal cord. This can be accomplished by disconnecting denticulate ligaments from the dura and rotating the spinal cord.

Another key component of tumor removal is the identification and division of proximal and distal nerve root tumor attachments. However, these attachments may not be apparent with large tumors, and intracapsular resection with suction or an ultrasonic aspirator is used in such cases. Sacrifice of the nerve root of origin is usually required for tumor removal after neurophysiological monitoring. More detailed information about the manipulation of nerve root attachments during surgery will be discussed in the following section.

\section{Surgical Strategy for Cervical Dumbbell- shaped Spinal Neurinomas}

\section{Classification of cervical dumbbell-shaped spinal neurinomas}

Dumbbell-shaped tumors comprise $20 \%$ of spinal neurinomas, and assume an hourglass shape owing to constriction at the point of penetration. This point typically occurs through the intervertebral foramen, where tumors are situated adjacent to the vertebral artery in the cervical region. These dumbbell-shaped spinal neurinomas are thought to form across the dura mater as a result of extradural growth along the nerve roots. ${ }^{13)}$

Eden's classification for dumbbell tumors of the spine, longtime considered a "gold standard," is no longer sufficient to determine surgical strategy in view of recent advances in neuroimaging. Asazuma et al. reported a new system to identify optimal surgical strategies for cervical dumbbell-shaped spinal neurinomas classified based on their shape and threedimensional location on diagnostic MR images. ${ }^{14,15)}$ Their classification includes nine main axially determined groups (Fig. 1). Type I comprises intra and extradural tumors that are located only in the spinal canal and constrict only the dura. Type II comprises epidural tumors with constriction at the foramen, and includes three subtypes (a, b, and c) defined according to the degree of extraforaminal spread. Type IIa tumors do not extend beyond the intervertebral foramen, while types IIb and IIc extend increasingly beyond the intervertebral foramen. Thus, IIa is extradural and foraminal; IIb, extradural and paravertebral; and IIc foraminal and paravertebral. Type III tumors have both dural and foraminal constriction, and include an intradural and extradural-foraminal type (type IIIa) and an intradural and extradural-paravertebral type (IIIb). Type IV tumors are extradural and intravertebral, invading only the vertebral body. Type $\mathrm{V}$ lesions are extradural and extralaminal with invasion of the lamina, and type VI tumors show multidirectional erosion of the bone.

This classification is based on the anatomical landmarks such as dura mater and intervertebral foramen. C1 and C2 vertebral body have no intervertebral foramina. Therefore, this is used for identification of optimal surgical strategy for cervical dumbbell-shaped spinal neurinomas located below C3 region.

\section{Surgical strategy for cervical dumbbell-shaped spinal neurinomas located in the upper cervical spine: $\mathrm{O}-\mathrm{C} 2$ region}

Many dumbbell-shaped neurinomas are found in the upper cervical spine. ${ }^{2)}$ These tumors, which have an extradural component, have been reported to decrease in number from the rostral to caudal region of the spinal axis. This growth pattern may be explained by anatomical features of the spinal nerve roots, which have a shorter intradural component at the rostral portion of the spinal canal. ${ }^{16-19)}$

Because the spinal canal is wide, compression of the spinal cord may be mild until tumors become large and dumbbell-shaped. Anatomically, as there are no intervertebral foramina and no osseous surrounding structures, the tumors tend to extend posterolaterally.

For upper cervical dumbbell-shaped spinal neurinomas such as those in the $\mathrm{C} 2$ region, the posterior approach is utilized because of the direction of the extradural component. ${ }^{20)}$ As a first step, the 


\section{Type I}
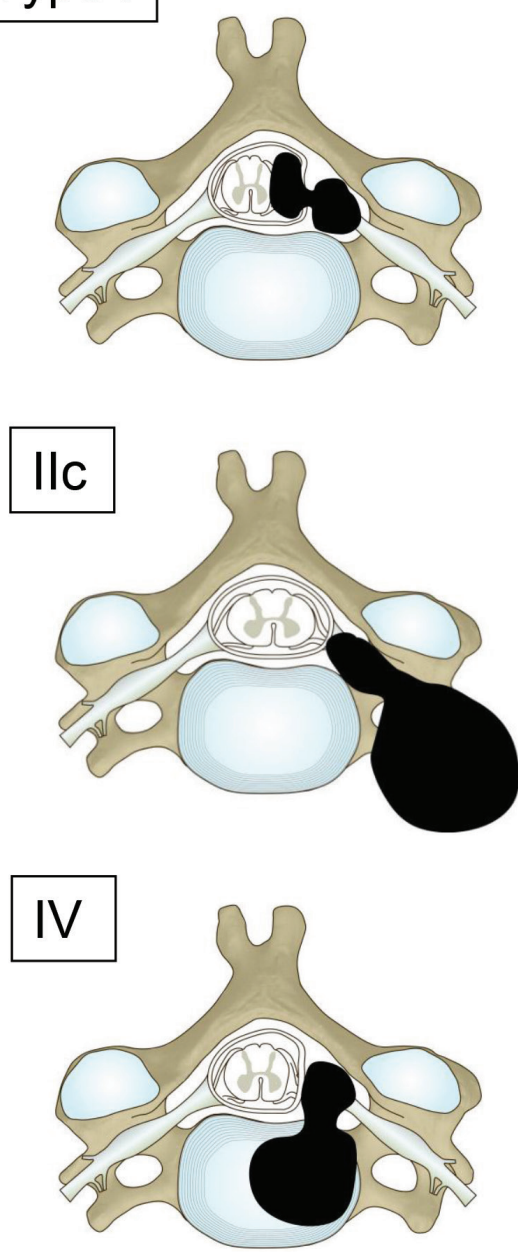
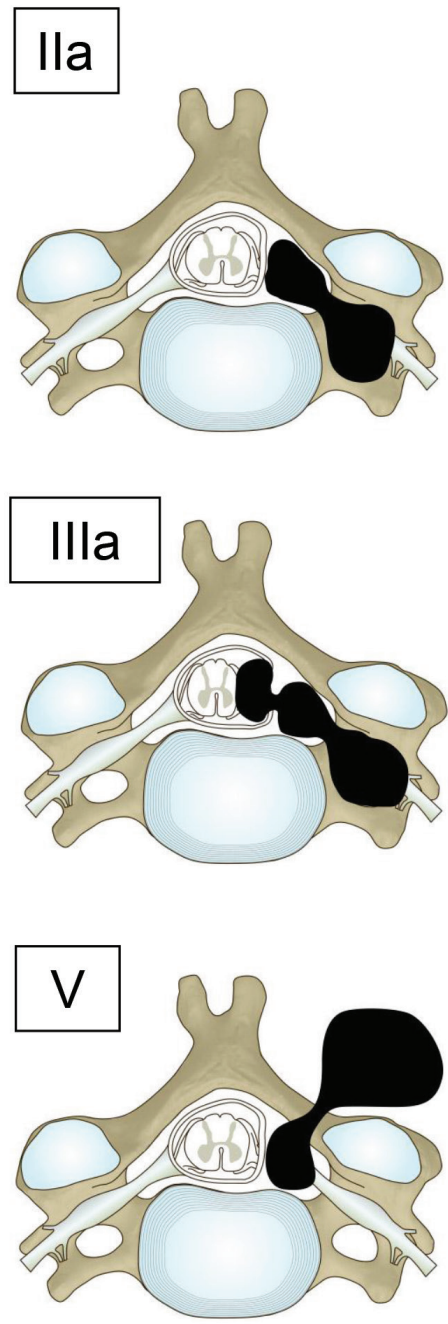
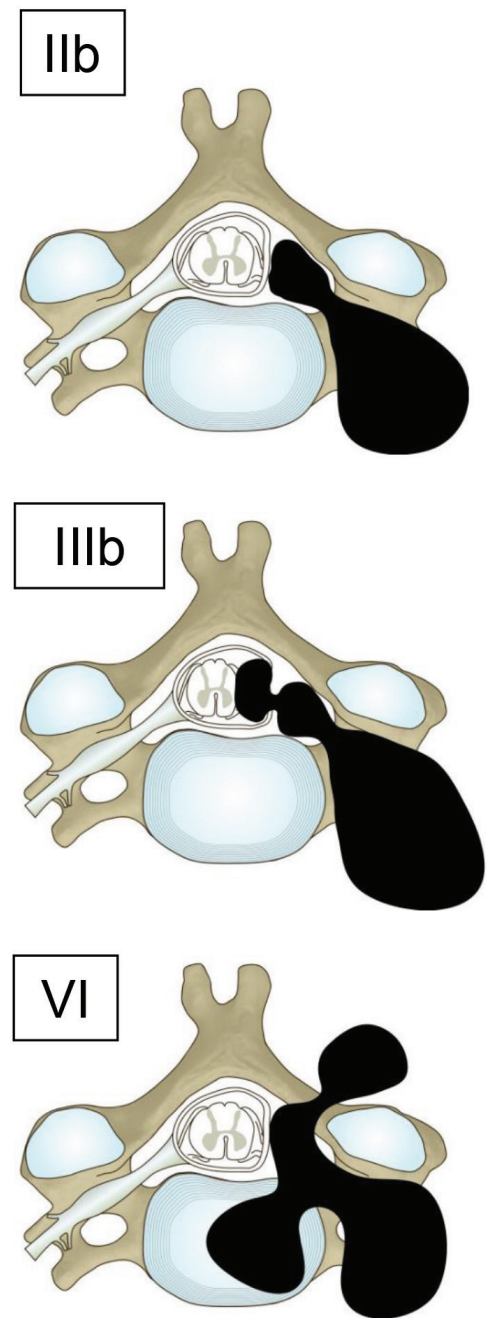

Fig. 1 Schematic drawing showing the Toyama classification for determining surgical strategy in the removal of dumbbell-shaped neurinomas. Type I, intradural and extradural tumors, located only in the spinal canal and which constrict only the dura. Type II, epidural tumors with constriction at the foramen. These include three subtypes ( $a, b$, and c) defined according to the degree of extraforaminal spread. IIa, extradural and foraminal; IIb, extradural and paravertebral; IIc, foraminal and paravertebral. Type III tumors, with both dural and foraminal constriction, include an intradural and extradural-foraminal type (type IIIa) and an intradural and extraduralparavertebral type (IIIb). Type IV tumors are extradural and intravertebral, and invade only the vertebral body. Type V lesions are extradural and extralaminal with invasion of the lamina. Type VI tumors show multidirectional erosion of the bone. (Reproduced from reference 17.)

extradural component of the tumor is removed in order to obtain a large enough operative field so that the tumor's feeding artery can be cut. A dural incision is then made along the extradural component, and if the tumor is preoperatively anticipated to adhere to the vertebral artery, an intracapsular resection is performed to prevent vertebral artery injury. Next, a longitudinal durotomy is made perpendicular to the previous incision so that the tumor's intradural component can be removed. This is done to obtain a wide operative field and cut feeding arteries to the tumor. In the case of a ventrally located tumor in the upper cervical spine, total removal is possible and is dependent on spinal cord rotation and the mobility of the tumor. On the other hand, the intraspinal component is removed first to prevent the compression to the spinal cord during surgery. ${ }^{21,22)}$ For large intradural tumors, a longitudinal paramedian durotomy that is gently curved laterally at either end is used. After intradural tumor removal, the dural incision is extended laterally over the nerve root sleeve. An intracapsular tumor dissection is performed if root preservation is possible. ${ }^{22)}$ 
III. Surgical strategy for cervical dumbbell-shaped spinal neurinomas located in the middle and lower cervical spine: $\mathrm{C} 2-\mathrm{C} 7$ region

The surgical strategy for a neurinoma located in the middle or lower cervical spine is more complicated, and selection of the most appropriate approach remains controversial. We have often encountered difficulties with complete resection of dumbbell-shaped tumors extending into the intervertebral foramen of the mid and lower cervical spine. In the literature, others have reported their surgical approaches in these more problematic cases, and they have also discussed surgery-related complications. ${ }^{5,23-28)}$ The literature, along with our experience, indicates that total removal of neurinomas is an achievable goal that yields good results; however, cervical dumbbellshaped neurinomas often present challenges in terms of complete resection. For complete removal, some dumbbell-shaped tumors require extensive surgical maneuvers, such as vertebrectomy. While several of these extensive approaches have been reported (Table 1), these procedures can lead to severe complications, such as arachnoiditis, ${ }^{29)}$ vertebral deformities, and vertebral artery injury. ${ }^{30)}$ Using the anterior or lateral approach with a total facetectomy, complete tumor resection has been described. ${ }^{31,32)}$ Moreover, Lot and George reported that complete resection could be achieved using a lateral approach with vertebral artery ligation. ${ }^{27)}$

Asazuma et al. reported a combined anterior and posterior approach is used for tumors that extend beyond the intervertebral foramen: types IIb, IIc, IIIb, and VI (Fig. 1). On the other hand, a posterior approach with partial or complete facetectomy can be employed in all other tumor type classifications. The results of 42 cases treated by this classification have been reported. With regard to radicality of resection, subtotal tumor resection has been performed in 6 patients $(14.3 \%)$, while total resection has been performed in 36 patients (85.7\%).

Table 1 Previous reports on surgical outcomes for cervical dumbbell-shaped neurinomas

\begin{tabular}{lcccc}
\hline Author & $\begin{array}{r}\text { Number } \\
\text { removal } \\
\text { rate (\%) }\end{array}$ & $\begin{array}{c}\text { Complication } \\
\text { rate (\%) }\end{array}$ & $\begin{array}{c}\text { Recurrence } \\
\text { rate (\%) }\end{array}$ \\
\hline $\begin{array}{l}\text { Klekamp } \\
\text { and Samii }\end{array}$ & 17 & 89 & 16.7 & 10.7 \\
$\begin{array}{l}\text { Jiang } \\
\text { et al. }\end{array}$ & 46 & 100 & 54.5 & 2.8 \\
$\begin{array}{l}\text { Nakamura } \\
\text { et al. }\end{array}$ & 75 & 76 & 18.6 & 5.3 \\
$\begin{array}{l}\text { Our case } \\
\text { Our) }\end{array}$ & 12 & 50 & 8.4 & 8.4 \\
\hline
\end{tabular}

In the six patients who underwent subtotal resection, two tumors were type IIa while four tumors were type IIIa. All the six tumors were foraminal and treated with a posterior approach. Moreover, there were no crucial intraoperative complications; thus, the classifications proposed by Asazuma et al. are thought to be clear and suitable. However, detailed long-term follow-ups of the remnant tumors have not been reported. Additionally, there are limitations to removing tumors located in the foraminal part of the spinal cord via a posterior approach.

\section{Recurrence rate of the tumor and postopera- tive complications}

As for the recurrence rate of cervical dumbbellshaped spinal neurinomas, Klekamp et al. noted a recurrence rate of $10.7 \%$ at 5 years. ${ }^{6}{ }^{6}$ Their surgical procedure included the removal of the facet joint to achieve complete resection of the extracanalicular portion of the tumor. However, in their report, complications occurred in $16.7 \%$ of the surgeries: one patient with infection, two with CSF leakage, one with hematoma, one with aseptic meningitis, and one with spinal instability. These complications might have resulted from the extensiveness of their surgical interventions. On the other hand, Jiang et al. reported a case series of 46 patients treated with gross total resection over a period of 8 years. ${ }^{25)}$ Interestingly, the recurrence rate for this series was $2.8 \%$ over an average 5 -year follow-up. It should be noted that that more extensive surgical techniques were applied in these cases, such as combined anterior and posterior approaches for total resection of the tumor that resulted in an average estimated blood loss of $1,650 \mathrm{ml}$. Because of this, their complication rate was high: $54.5 \%$ of surgeries.

Little is known about the natural behavior of neurinomas, particularly of postoperative tumor remnants. Therefore, the surgical strategy for dumbbell-shaped neurinomas is controversial. Previously, we devised a study to assess the surgical outcomes and chronological changes of remnant dumbbell-shaped neurinomas following a posterior approach for surgical resection. ${ }^{23)}$ Twelve patients with dumbbell-shaped neurinomas were surgically treated at our institute from 2004 to 2011. We adopted a posterior approach to remove the intracanalicular portion of the neurinomas as completely as possible, intentionally leaving the foraminal portion unresected to prevent bony and vascular injury. Postoperative residual tumors were identified in 6 of the 12 patients, and the follow-up period ranged from 12 months to 88 (mean $65.3 \pm$ 19.9) months. Perioperative neurological findings between the dumbbell-shaped subtotal or partial removed group and dumbbell-shaped total removed 
group were almost the same, and we found that the recurrence rate was $8.4 \%$ of all dumbbell-shaped neurinoma cases. Follow-up MR images showed no enlargement of the remnant tumors except in one patient with a cystic component, which are known to grow easily. ${ }^{23)}$ Moreover, surgery-related complications occurred only in one patient with mild and temporary motor weakness. Taken together, our strategy demonstrates lower rates of surgical complications and similar rate of tumor recurrence compared with those accompanying extensive alternative approaches.

Nakamura et al. reported another treatment strategy for cervical dumbbell neurinomas. ${ }^{33)}$ Of the 18 cases where tumors had been partially resected (among 75 cases of cervical dumbbell neurinoma reviewed), only 4 cases demonstrating high tumor-growth rates required re-operation or showed tumor growth. Among the four re-operated cases, the MIB-1 index, which was $5 \%$ and $8 \%$, respectively in the two cases, was high. These cases emphasize that total resection should be attempted for cervical dumbbell tumors. However, in cases where total resection poses a potentially high risk, subtotal resection can be a practical choice yielding favorable long-term outcomes.

Sohn et al. evaluated the growth rate of residual spinal schwannomas using data collected from the Korea Spinal Oncology Research Group database. ${ }^{34)}$ More specifically, they compared groups of patients who had experienced tumor regrowth to those who did not exhibit regrowth. Age, sex, tumor size at initial diagnosis, postoperative tumor size, and MIB-1 index were compared between the two groups. Interestingly, only MIB-1 indices were significantly different between regrowth and no regrowth groups (6.3 \pm 5.6 vs. $2.0 \pm 1.6 \%, P=0.014)$. They postulated that the remnant tumors with high MIB-1 indices should be done under close observation.

\section{Problems Associated with Treatment of Spinal Neurinomas}

\section{Postoperative neurological dysfunction due to sacrifice the nerve root of tumor origin}

Cases where the entire nerve root is involved are often encountered with dumbbell-shaped neurinomas in which the tumor is large, and has progressed extradurally. Such cases often lead to concerns about the occurrence of postoperative dysfunction. Kim et al. reported their results as follows: (1) in $22.6 \%$ of cases, temporary motor and sensory dysfunction appeared without serious complications, (2) given their histological properties, neurofibromas are likely to cause neurological disorders after excision, but in practice, there have been no reports of postoperative neuropathy, and (3) similar to dumbbell-shaped spinal neurinomas, no differences in tumor size or incidence of postoperative neurological deficits were observed with relatively large tumors. Ultimately, the authors concluded that the nerve root from which the tumor originated lost its function during tumor removal and that other nerves can compensate for the loss of function. As a result, the postoperative neurological deficit is mild, even when the origin is excised along with the tumor during surgery. ${ }^{26)}$ Celli focused on the fact that severe mobility disorders, although uncommon ( $7.4 \%$ in incidence), did appear after surgery, and that such disorders were more likely to occur with tumors located extradurally. ${ }^{24)}$ Hasegawa et al. reported similar results from a histological study. The morphology of nerve roots from which tumors originate has been investigated using optical and electron microscopy. These studies revealed that nerve roots serving as origins have decreased myelinated nerve fibers. Notably, as degeneration occurs slowly, it is possible for surrounding nerves to compensate for functional activity. ${ }^{35)}$ Lot and George evaluated the presence or absence of denervation using electromyography in order to determine whether neurological symptoms appeared consequent to nerve transection during surgery. According to the results of their observations, they decided to sacrifice nerve roots in $28 \%$ of extradural schwannoma cases. Impressively, only $3.5 \%$ of the cases exhibited postoperative motor weakness. Due to these results, functional nerve root assessment with intraoperative nerve stimulation has been advocated as a necessary procedure. ${ }^{27)}$

\section{Treatment strategy for neurinomas associated with NF type 2}

According to an analysis of 87 patients by Klekamp et al., outcomes of treatments for spinal neurinomas differed between patients with and without NF type 2. More specifically, in patients without NF type 2, $91 \%$ of tumors were removed and the patients' Karnofsky performance scores significantly improved from $70 \pm 13 \%$ preoperatively to $87 \pm 8 \%$ postoperatively. However, with NF type 2 cases, complete removal was achieved only in $74 \%$ of patients, and the pre- and postoperative Karnofsky performance scores showed no significant difference at $58 \pm$ $15 \%$ and $68 \pm 21 \%$, respectively. This difference in outcome was reported to be associated with the fact that many patients with NF type 2 had multiple large tumors extending into the epidural space. In addition, malignant neurinomas occur in $30-50 \%$ of patients with NF type $2 .^{31)}$ In contrast, it has been reported that there is no difference in preoperative 
neurological symptoms, surgical findings, surgical results, or recurrence rates between patients with and without NF type $1{ }^{6}{ }^{6}$

\section{Conclusion}

A cervical spinal neurinoma is a benign tumor that is common among the spinal cord tumors. However, while these tumors are relatively common, many issues need to be addressed in terms of their appropriate treatment. With advances in neuroimaging and neurophysiological monitoring, appropriate surgical approaches and intraoperative surgical manipulations should be chosen for each case.

\section{Acknowledgments}

In writing this review, the authors express their gratitude to their colleagues in the Department of Neurosurgery, Shinshu University School of Medicine, for their daily contributions to patients with spinal neurinoma.

\section{Conflicts of Interest Disclosure}

The authors have made declarations of their conflicts of interest every year to the Japan Neurosurgical Society. They have no personal, financial, or institutional relationships with other individuals or organizations that could inappropriately influence this work.

\section{References}

1) Klekamp J, Samii M: Introduction of a score system for the clinical evaluation of patients with spinal processes. Acta Neurochir (Wien) 123: 221-223, 1993

2) Jinnai T, Koyama T: Clinical characteristics of spinal nerve sheath tumors: analysis of 149 cases. Neurosurgery 56: 510-515; discussion 510-515, 2005

3) George B, Lot G: Neurinomas of the first two cervical nerve roots: a series of 42 cases. J Neurosurg 82: 917-923, 1995

4) Pilavaki M, Chourmouzi D, Kiziridou A, Skordalaki A, Zarampoukas T, Drevelengas A: Imaging of peripheral nerve sheath tumors with pathologic correlation: pictorial review. Eur J Radiol 52: 229-239, 2004

5) Skovronsky DM, Oberholtzer JC: Pathologic classification of peripheral nerve tumors. Neurosurg Clin N Am 15: 157-166, 2004

6) Klekamp J, Samii M: Surgery of spinal nerve sheath tumors with special reference to neurofibromatosis. Neurosurgery 42: 279-289; discussion 289-290, 1998

7) Ito K, Ishizaka S, Sasaki T, Miyahara T, Horiuchi T, Sakai K, Shigeta H, Hongo K: Safe and minimally invasive laminoplastic laminotomy using an ultrasonic bone curette for spinal surgery: technical note. Surg Neurol 72: 470-475; discussion 475, 2009

8) Herman JM, Sonntag VK: Cervical corpectomy and plate fixation for postoperative kyphosis. J Neurosurg 80: 963-970, 1994

9) Hirabayashi K, Watanabe K, Wakano K, Suzuki N, Satomi K, Ishii Y: Expansive open-door laminoplasty for cervical stenotic myelopathy. Spine (Phila $\mathrm{Pa}$ 1976) 8: 693-699, 1983

10) Hirabayashi K, Satomi K: Operative procedure and results of expansive open-door laminoplasty. Spine (Phila Pa 1976) 13: 870-876, 1988

11) Itoh $\mathrm{T}$, Tsuji $\mathrm{H}$ : Technical improvements and results of laminoplasty for compressive myelopathy in the cervical spine. Spine (Phila Pa 1976) 10: 729-736, 1985

12) Steinmetz MP, Resnick DK: Cervical laminoplasty. Spine J 6(6 Suppl): 274S-281S, 2006

13) Ozawa H, Kokubun S, Aizawa $T$, Hoshikawa $T$, Kawahara C: Spinal dumbbell tumors: an analysis of a series of 118 cases. J Neurosurg Spine 7: 587-593, 2007

14) Asazuma T, Toyama $Y$, Maruiwa H, Fujimura $Y$, Hirabayashi K: Surgical strategy for cervical dumbbell tumors based on a three-dimensional classification. Spine (Phila Pa 1976) 29: E10-E14, 2004

15) Toyama Y, Fujimura Y, Takahata T: Clinical analysis of 83 cases of the dumbbell tumors in the spine: morphological classification and surgical management. J Jpn Med Soc Paraplegia 5: 86-87, 1992 (Japanese)

16) Mizuno J, Nakagawa H, Hashizume Y: Microsurgical anatomy of the entire cervical spine, in Mizuno J (ed): Surgical Anatomy for Microneurosurgery. Tokyo, Sci Med Publications, 1994, pp 3-10

17) Asazuma $\mathrm{T}$ : Combined anterior and posterior approaches for cervical dumbbell tumor. Spine \& Spinal Cord 21: 231-238, 2007

18) Seppälä MT, Haltia MJ, Sankila RJ, Jääskeläinen JE, Heiskanen O: Long-term outcome after removal of spinal schwannoma: a clinicopathological study of 187 cases. J Neurosurg 83: 621-626, 1995

19) Tanaka N, Fujimoto Y, An HS, Ikuta Y, Yasuda M: The anatomic relation among the nerve roots, intervertebral foramen, and intervertebral discs of the cervical spine. Spine (Phila Pa 1976) 25: 286-291, 2000

20) Uchikado $\mathrm{H}$ : Intradural extramedurally tumor, in Imae S, Kawanishi M, Asamoto S, Kawamoto (eds): Guidebook of Diagnosis and Treatment for Spinal Disease, ed 1. Tokyo, Medical View Co., Ltd. 2010, pp 182-183

21) Kim P: Extramedullary tumor, in Kim $\mathrm{P}$ (ed): $A$ Guidebook for the Future Experts in the Cervical Spine Surgery From Neurological Evaluation to Microsurgical Techniques, ed 1. Tokyo, Medical View Co., Ltd., 2007, pp 230-240

22) McCormick PC: Surgical management of dumbbell tumors of the cervical spine. Neurosurgery 38: 294-300, 1996 
23) Ito K, Aoyama T, Kuroiwa M, Horiuchi T, Hongo $\mathrm{K}$ : Surgical strategy and results of treatment for dumbbell-shaped spinal neurinoma with a posterior approach. Br J Neurosurg 28: 324-329, 2014

24) Celli P: Treatment of relevant nerve roots involved in nerve sheath tumors: removal or preservation? Neurosurgery 51: 684-692; discussion 692, 2002

25) Jiang L, Lv Y, Liu XG, Ma QJ, Wei F, Dang GT, Liu ZJ: Results of surgical treatment of cervical dumbbell tumors: surgical approach and development of an anatomic classification system. Spine (Phila $\mathrm{Pa}$ 1976) 34: 1307-1314, 2009

26) Kim P, Ebersold MJ, Onofrio BM, Quast LM: Surgery of spinal nerve schwannoma. Risk of neurological deficit after resection of involved root. J Neurosurg 71: 810-814, 1989

27) Lot G, George B: Cervical neuromas with extradural components: surgical management in a series of 57 patients. Neurosurgery 41: 813-820; discussion 820-822, 1997

28) Medin PM, Boike TP: Spinal cord tolerance in the age of spinal radiosurgery: lessons from preclinical studies. Int J Radiat Oncol Biol Phys 79: 1302-1309, 2011

29) O’Connor M, Brighouse D, Glynn CJ: Unusual complications of the treatment of chronic spinal arachnoiditis. Clin J Pain 6: 240-242, 1990

30) Yasuoka S, Peterson HA, MacCarty CS: Incidence of spinal column deformity after multilevel laminectomy in children and adults. J Neurosurg 57: 441-445, 1982
31) Conti P, Pansini G, Mouchaty H, Capuano C, Conti R: Spinal neurinomas: retrospective analysis and long-term outcome of 179 consecutively operated cases and review of the literature. Surg Neurol 61: 34-43; discussion 44, 2004

32) McCormick PC: Surgical management of dumbbell tumors of the cervical spine. Neurosurgery 38: 294-300, 1996

33) Nakamura M, Iwanami A, Tsuji O, Hosogane N, Watanabe K, Tsuji T, Ishii K, Toyama Y, Chiba $\mathrm{K}$, Matsumoto M: Long-term surgical outcomes of cervical dumbbell neurinomas. J Orthop Sci 18: 8-13, 2013

34) Sohn S, Chung CK, Park SH, Kim ES, Kim KJ, Kim $\mathrm{CH}$ : The fate of spinal schwannomas following subtotal resection: a retrospective multicenter study by the Korea spinal oncology research group. J Neurooncol 114: 345-351, 2013

35) Hasegawa M, Fujisawa H, Hayashi Y, Tachibana O, Kida S, Yamashita J: Surgical pathology of spinal schwannoma: has the nerve of its origin been preserved or already degenerated during tumor growth? Clin Neuropathol 24: 19-25, 2005

Address reprint requests to: Kiyoshi Ito, $\mathrm{MD}, \mathrm{PhD}$, Department of Neurosurgery, Shinshu University School of Medicine, 3-1-1 Asahi, Matsumoto, Nagano 390-8621, Japan.

e-mail: kitoh@shinshu-u.ac.jp 